\title{
Radical Young's Diagrams Core
}

\section{Ammar S. Mahmood}

Hanan S. Mohammed

Department of Mathematics / College of Education

University of Mosul

Received

27 / 05 / 2009
Accepted

$16 / 02 / 2010$

الخلاصة:

هذا البهث هو أسلساً في موضوع ظارية التمثل Representation theory وتحديداً

في جبرشور من النوع q-Schur algebra) q) وجبر هيكا (Hecke algebra) حيث تلعب البه

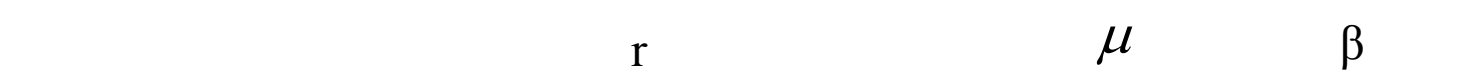

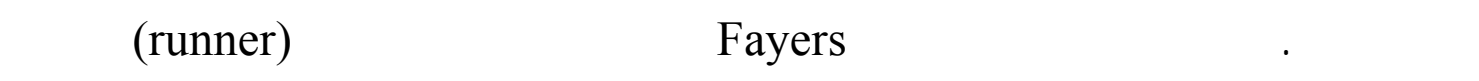

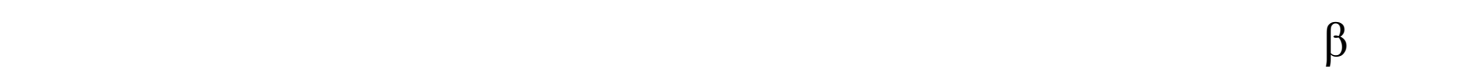

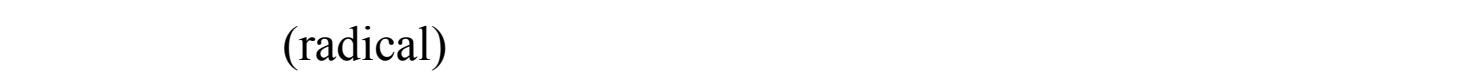
رياضياً وبرمجياً.

\section{Abstract:}

This is research is basically deals with the representation theory that is specifically on Hecke algebra and q-Schur algebra, where $\beta$ numbers of a partition $\mu$ has sufficient effect in both types of algebra.

The objective of this work is to expand the results of Fayers that is by adding new runners to $\beta$-numbers, to represent a "tree", and by other hand, we decide to reduce the runners reaching another new definition "radical" by using both mathematically and computer programming ways.

Keywords: Hecke algebra, $q$-Schur algebra, $\beta$-numbers and e- Core

\section{Introduction:}

Let $\mathrm{F}$ be a field, $\mathrm{q}$ an invertible element of $\mathrm{F}, \mathrm{r}$ a non-negative integer and $G_{r}$ a symmetric group. We define $e>1$ to be minimal such that $1+\mathrm{q}+\ldots . .+\mathrm{q}^{\mathrm{e}-1}=0$, with $\mathrm{e}=\infty$ if no such integer exists, then we shall assume that $\mathrm{e}$ is finite.

A composition $\mu$ of $\mathrm{r}$ is a sequence $\left(\mu_{1}, \mu_{2}, \ldots, \mu_{n}\right)$ of non-negative integers such that $|\mu|=\sum_{i=1}^{n} \mu_{i}=\mathrm{r}$. A composition $\mu$ is a partition if 
$\mu_{i} \geq \mu_{i+1}$ for all $\mathrm{i} \geq 1$. The diagram of Young of a composition $\mu$ is the subset :

$$
[\mu]=\left\{(\mathrm{x}, \mathrm{y}) \mid 1 \leq y \leq \mu_{x} \text { and } x \geq 1\right\} \text { of } N \times N,
$$

it is useful to represent the diagram of $\mu$ as an array of boxes in the plane, for example, if $\mu=(2,3)$ then $[\mu]$

We denote the $\mu$-composition of $\mathrm{r}$ as $\mu$ Fr and denote the $\mu$ partition of $\mathrm{r}$ as $\mu \mathrm{Fr}$. The best introduction to the representation theory of Iwahori- Hecke algebra and q-Schur algebra can be found in Mathas's book [9], as the following: Let $\mathrm{H}_{\mathrm{r}}=\mathrm{H}_{\mathrm{F}, \mathrm{q}}(\mathrm{r})$ be the Iwahori-Hecke algebra of $\mathrm{G}_{\mathrm{r}}$ and let $\mathrm{S}_{\mathrm{q}}(\mathrm{n}, \mathrm{r})$ be the corresponding q-Schur algebra. $\mathrm{H}_{\mathrm{r}}$ is the associative F-algebra with basis $\left\{T_{W} \mid \mathrm{W} \in \mathrm{G}_{\mathrm{r}}\right\}$ and multiplication determined by:

$$
\mathrm{T}_{\mathrm{S}_{\mathrm{i}}} \mathrm{T}_{\mathrm{W}}=\left\{\begin{array}{lc}
\mathrm{T}_{\mathrm{S}_{\mathrm{i}} \mathrm{W}} & \text { if } \mathrm{i}^{\mathrm{w}}<(\mathrm{i}+1)^{\mathrm{w}} \\
\mathrm{qT}_{\mathrm{S}_{\mathrm{w}}}+(\mathrm{q}-1) \mathrm{T}_{\mathrm{w}} & \text { otherwise }
\end{array}\right.
$$

where $w \in G_{r}$ and $s_{i}=(i, i+1)$, for $i=1,2,3, \ldots, r-1$. The q-Schur algebra is the endomorphism algebra

$$
\mathrm{S}_{\mathrm{q}}(\mathrm{n}, \mathrm{r})=\text { End }_{\mathrm{H}_{\mathrm{r}}}\left(\underset{\mu \mathrm{r}}{\oplus} x_{\mu} \mathrm{H}_{\mathrm{r}}\right)
$$

where $x_{\mu}=\sum_{\mathrm{w} \in \mathrm{G}_{\mathrm{r}}} \mathrm{T}_{w}$ and a Young subgroup

$$
\mathrm{G}_{\mu}=\mathrm{G}_{\mu_{1}} \times \mathrm{G}_{\mu_{2}} \times \ldots \ldots . . \times \mathrm{G}_{\mu_{n}} \quad \text { of } \mathrm{G}_{r} .
$$

Dipper and James in [1] defined the Specht module $S^{\mu}$; for each partition $\mu$ of $\mathrm{r}$ there is a right $\mathrm{H}_{\mathrm{r}}$-module $S^{\mu}$. A partition $\mu$ is $e$-regular if it does not have $e$ non-zero equal parts. If $\mu$ is $e$-regular then $S^{\mu}$ has an irreducible cosocle $D^{\mu}$. Also, a Weyl module $\mathrm{W}^{\mu}$ is defined as, for any partition $\mu$ of $\mathrm{r}$, there is a right $\mathrm{S}_{\mathrm{q}}(\mathrm{n}, \mathrm{r})$-module $\mathrm{W}^{\mu}$. The $\operatorname{cosocle} L^{\mu}$ of $\mathrm{W}^{\mu}$ is irreducible.

Given partitions $\mu$ and $\lambda$ of $\mathrm{r}$, with $e$-regular, let $\left\lfloor S^{\mu}: D^{\lambda}\right\rfloor$ be the multiplicity of $D^{\lambda}$ as a composition factor of $S^{\mu}$. Similarly, let $\left[\mathrm{w}^{\mu}: L^{\lambda}\right]$ be the multiplicity of $L^{\lambda}$ as a composition factor of $\mathrm{W}^{\mu}$. With $\mu$ is e-regular, $\left(\left[S^{\mu}: D^{\lambda}\right]\right)_{\mu, \lambda \vdash \mathrm{r}}$ is the decomposition matrix of $\mathrm{H}_{\mathrm{r}}$ and $\left(\left[\mathrm{W}^{\mu}: L^{\lambda}\right]\right) \mu, \lambda \vdash_{\mathrm{r}}$ is the decomposition matrix of $\mathrm{S}_{\mathrm{q}}(\mathrm{n}, \mathrm{r})$, see [8].

\section{2. $\beta$-numbers and e-Core :}

Choose an integer $b$ greater than the number of parts of a partition $\mu$, and define

$$
\boldsymbol{\beta}_{\mathbf{j}}=\mu_{\mathbf{j}}+\mathbf{b}-\mathbf{j} \text {, for } \mathbf{j}=1,2, \ldots . ., b \text {. }
$$

The set $\left\{\beta_{1}, \ldots, \beta_{\mathrm{b}}\right\}$ is said to be a set of beta-number for $\mu$. For example, if $\mu=\left(5,3^{2}, 2,1\right)$, then the number of parts of $\mu$ is 5 . Let $b=7$, then $\beta$ numbers are $(11,8,7,5,3,1,0)$. 
We consider an abacus with e vertical runners, labeled $0,1, \ldots, \mathrm{e}-1$ from left to right. And label the partition on runner $j$ as $j, j+e, j+2 e, \ldots$. from the top downwards. We call the bead position me, me $+1, \ldots$, me $+\mathrm{e}-1$ row $\mathrm{m}$ of the abacus configuration for $\mu$ with $\mathrm{b}$ beads is the abacus configuration obtained by placing a bead at position $\beta_{\mathrm{j}}$ for $\mathrm{j}=1,2, \ldots, \mathrm{b}$.

$\begin{array}{ccccc}0 & 1 & 2 & \ldots & \mathrm{e}-1 \\ \mathrm{e} & \mathrm{e}+1 & \mathrm{e}+2 & \ldots & 2 \mathrm{e}-1 \\ 2 \mathrm{e} & 2 \mathrm{e}+1 & 2 \mathrm{e}+2 & \ldots & 3 \mathrm{e}-1\end{array}$

From the above example, if $\mathrm{e}=2$,

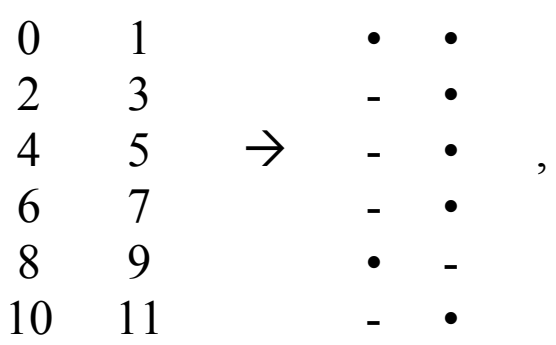

$\mathrm{e}=3$,

$\begin{array}{ccc}0 & 1 & 2 \\ 3 & 4 & 5 \\ 6 & 7 & 8 \\ 9 & 10 & 11\end{array}$

and if $\mathrm{e}=4$, then

$\begin{array}{cccc}0 & 1 & 2 & 3 \\ 4 & 5 & 6 & 7 \\ 8 & 9 & 10 & 11\end{array}$

Given an abacus configuration for $\mu$ we can create a new abacus configuration by moving all beads as high as possible on each runner. The partition; denoted by $\rho$, corresponding to this new abacus configuration is called the e-core of $\mu$,

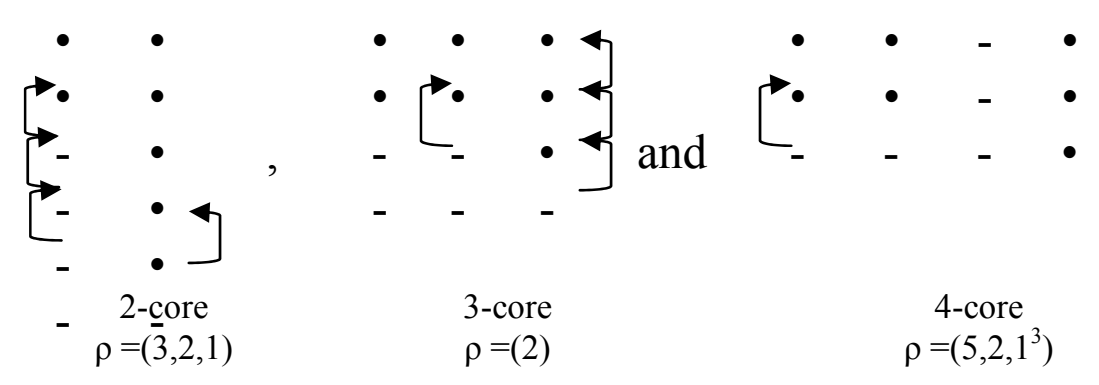


Rule (2.1): We can find an easy rule for finding any partition of any ecore and as follows:

"first we count the spaces in all runners before the last bead, which is equal to $\rho_{1}$. Then we subtract the spaces from $\rho_{1}$ for the last bead with the one before the last, the result would be denoted as $\rho_{2}$. This procedure will be repeated on $\rho_{2}$, that is to subtract the spaces from $\rho_{2}$ for the bead before the last with the one before it, and denoted by $\rho_{3}$, and so on ....".

Theorem (2.2) : [7]

Each partition has a uniquely e-core.

If $\rho$ is the e-core of $\mu$ then e-weight of $\mu$ is: $w=\frac{|\mu|-|\rho|}{\mathrm{e}}$, for the above example,

$\mu=\left(5,3^{2}, 2,1\right) \Rightarrow|\mu|=14, \quad|\rho|_{e=2}=6, \quad|\rho|_{e=3}=2$ and $|\rho|_{e=4}=10$.

Then $\quad \mathrm{W}_{\mathrm{e}=2}=\frac{14-6}{2}=4, \quad \mathrm{~W}_{\mathrm{e}=3}=\frac{14-2}{3}=4 \quad$ and $\quad \mathrm{W}_{\mathrm{e}=4}=\frac{14-10}{4}=1$.

For more application with e-weight, see [2], [5] and [6].

The definition of e-weight is equivalent exactly to e-quotient; see [7]:

"We write $\mu_{b}^{a}$ for the number of unoccupied positions above the bth lowest bead on runner a", then $\mu(a)=\left(\mu_{1}^{a}, \mu_{2}^{a}, \ldots.\right)$ is a partition, and we refer to the sequence $(\mu(0), \ldots . ., \mu(e-1))$ as the e-quotient of $\mu$.

Then we have ((3), (1)), ((0), (1), (1 $\left.\left.{ }^{3}\right)\right)$ and $((1),(0),(0),(0))$ if we use $\mathrm{e}=2, \mathrm{e}=3$ and $\mathrm{e}=4$ respectively.

According to the beads, we can find many different cases having the same weight for $\mu$ but also having the same core. These cases can be shown from the last example and achieve the aim:

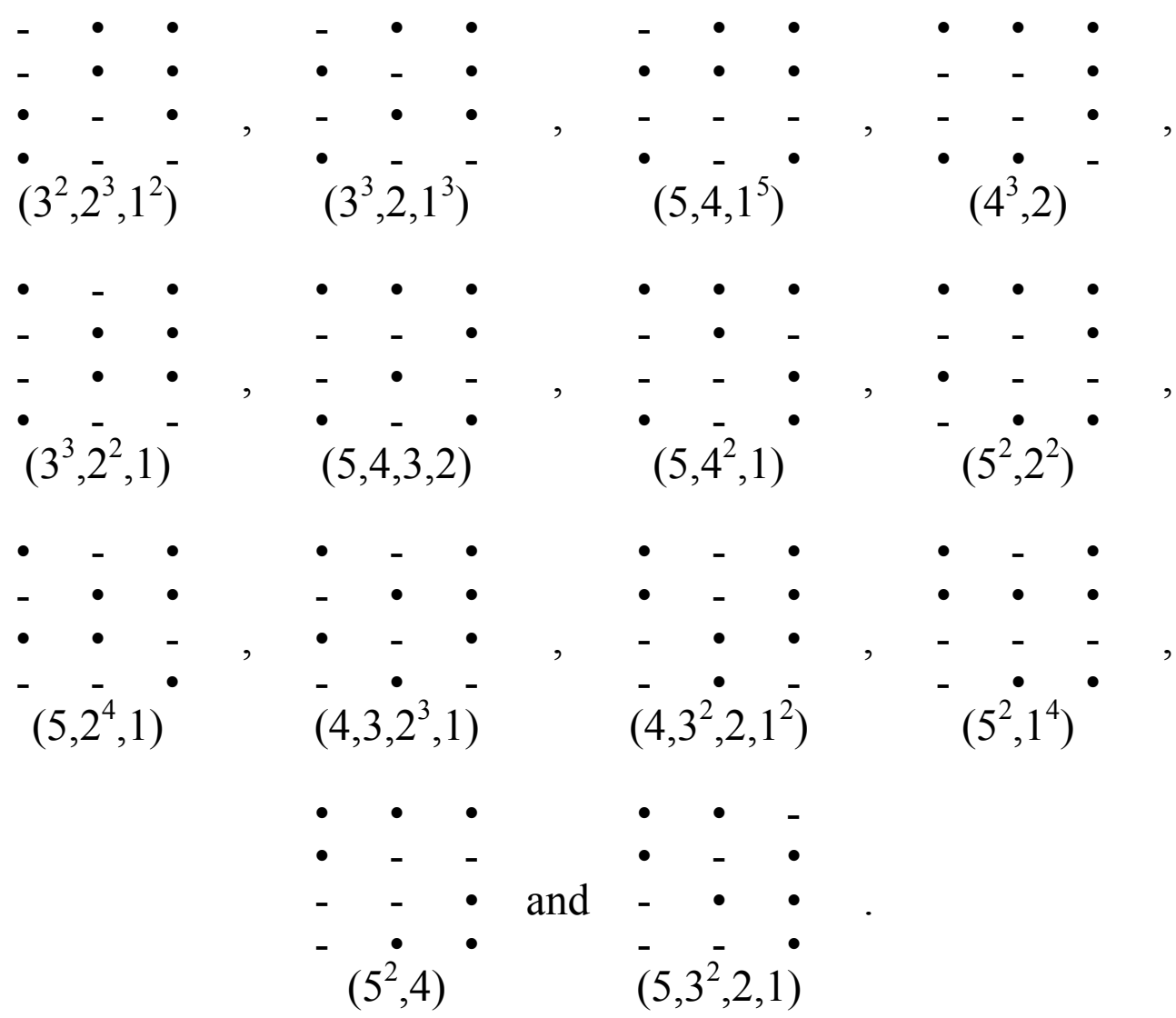




\section{Theorem (2.3) "Nakayama conjecture": [9]}

The two modules of Weyl $\mathrm{W}^{\mu}$ and $\mathrm{W}^{\lambda}$ belong to the same block if and only if $\mu$ and $\lambda$ have the same weight and the same core, similarly, for two modules of Specht $s^{\mu}$ and $s^{\lambda}$.

Rule (2.4): The maximum weight $\left(\max _{\mathrm{w}}\right)$ can be calculated and found for any core which is equal to the sum of all products the number of beads by the number of spaces of the same runner.

As a result, the $\max _{\mathrm{w}}$ for the case $\mu=\left(5,3^{2}, 2,1\right)$ and $\mathrm{e}=3$ is $(2 \times 2)+(2 \times 2)+(3 \times 1)=11$, where $\max _{\mathrm{w}}=4$ when $\mathrm{e}=4$.

\section{Trees and Radicals :}

According to Fayers in [4], who was able to make an easy way to insert one runner to the $\beta$-numbers by putting number of beads under consideration that the last bead location in this runner does not exceed the location of $\beta_{1}$ but left with a space, otherwise, if this bead exceeds $\beta_{1}$ without making a space, this case will be calculated by using Fayers research of this insertion as follows:

"Given a partition $\mu$ and a non-negative $\mathrm{K}$, we constrict a new partition $\mu^{\mathrm{tK}}$ as follows. Take $\mathrm{b} \geq \mu_{1}^{\prime}$ and constrict the abacus display for $\mu$ with $b$ beads. Write $b+K=c e+d$ with $0 \leq d \leq e-1$, and add a runner to the abacus display immediately to the left of runner $\mathrm{d}$; now put $\mathrm{c}$ beads on this new runner in the top c position. The partition whose abacus display is obtained, is $\mu^{+\mathrm{k} "}$.

By the pervious example, when $\mu=\left(5,3^{2}, 2,1\right)$, if we picked $\mathrm{e}=2$ then

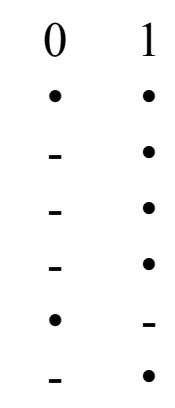


Therefore, we'll have the following cases for $\mu^{+\mathrm{K}}$ :

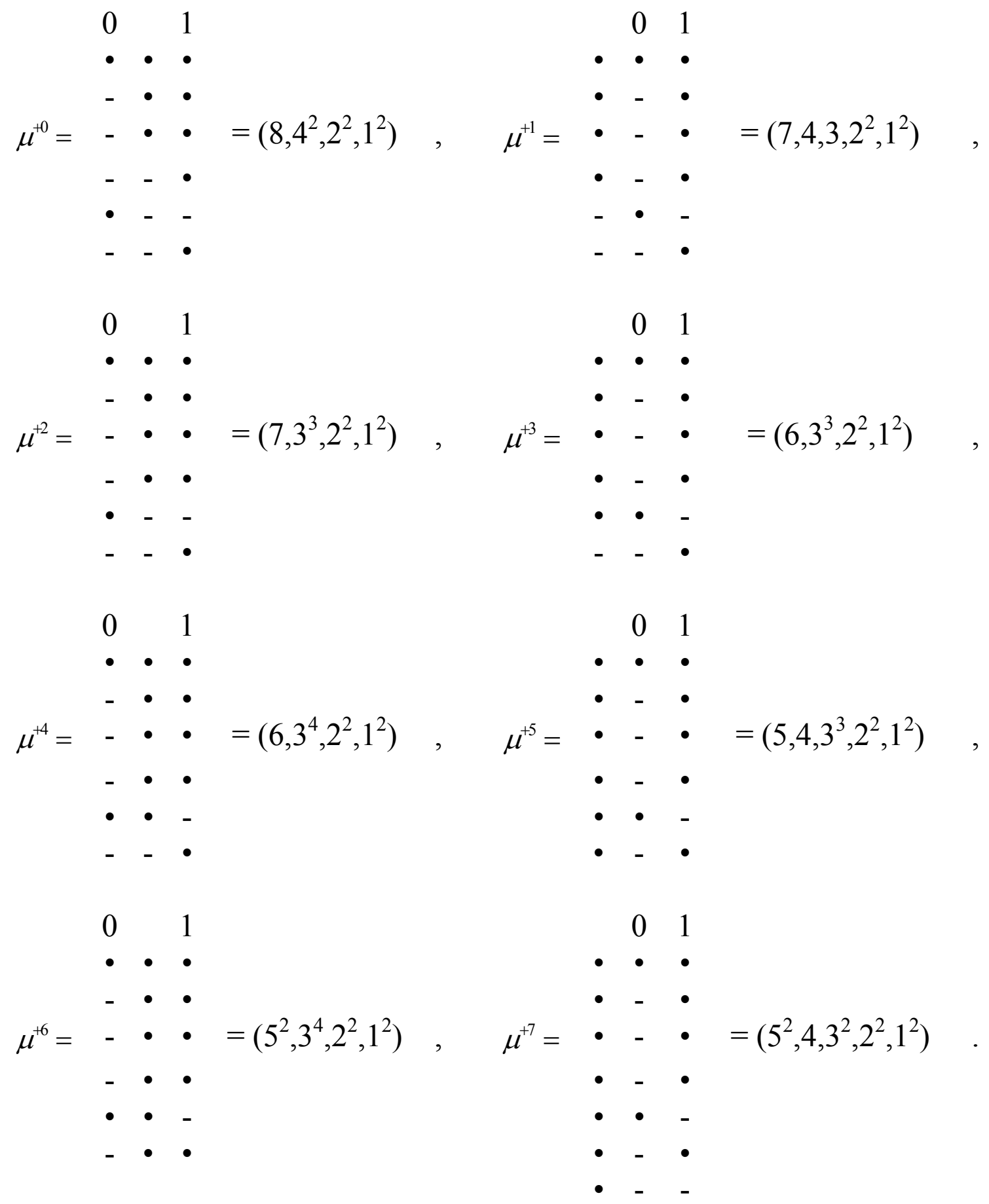

In this study we are able to insert many runners to an $\beta$-numbers and get $\mu^{+\mathrm{K},+\mathrm{K}^{\prime},+\mathrm{K}^{\prime \prime}, \ldots \ldots \ldots}$ which will from a " Tree " or " group of trees ".

\section{Rule (3.1):}

1) For choosing $+K$, we follow the previous steps offered by Fayers in [4].

2) For choosing $+K^{\prime}$, we'll depend on the value of 


$$
\begin{aligned}
\mathrm{b}^{\prime} & =\mathrm{b}+\mathrm{c} \\
& =\mathrm{b}+\frac{\mathrm{b}+\mathrm{K}-\mathrm{d}}{\mathrm{e}}
\end{aligned}
$$

under condition that $d$ is fit to the value of $b+K-d$ is divisible by e. Obviously, $\mathrm{e}^{\prime}=\mathrm{e}+1$, and the solution is continued using the same technique of Fayers.

3) Repeat the same way in $\mathrm{c}$ for the rest of steps by:

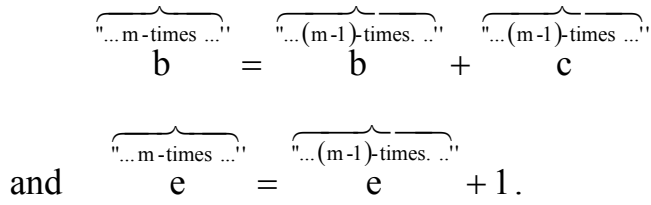

We denote these cases by trees.

For example, the following tree: $\mu^{+1,+1,+2}$ is the extension to $\mu^{+1}$ where $\mu=\left(5,3^{2}, 2,1\right)$ :

The following program is for finding this tree :

function

matrix=check_acception_matrix(may_be_matrix,accepted_x_position,acc epted_y_position)

matrix $=1$;

$\mathrm{mm}=\max ($ find $($ may_be_matrix $($ end,: $)==1))$;

if $\mathrm{mm}>$ accepted_y_position

matrix $=0$;

end

function insertion $1=$ ins_column $(\mathrm{a}, \mathrm{v}, \mathrm{k})$;

$\% / a /$ is the matrix that we must insert the first elements of vector $/ \mathrm{v} /$ in it before the

$\%$ position $/ \mathrm{k} /$ here the value of $/ \mathrm{k} /$ may be $\{0,1,2,3, \ldots, \operatorname{size}(\mathrm{a}, 2)-1\}$

$[\mathrm{m} \mathrm{n}]=\operatorname{size}(\mathrm{a})$;

$\mathrm{mm}=$ length $(\mathrm{v})$;

if $\mathrm{mm} \sim=\mathrm{m}$

' The dimension of the vector is not correct to put it in the matrix' $\% \#$ ok $<$ NOPRT $>$

return; 


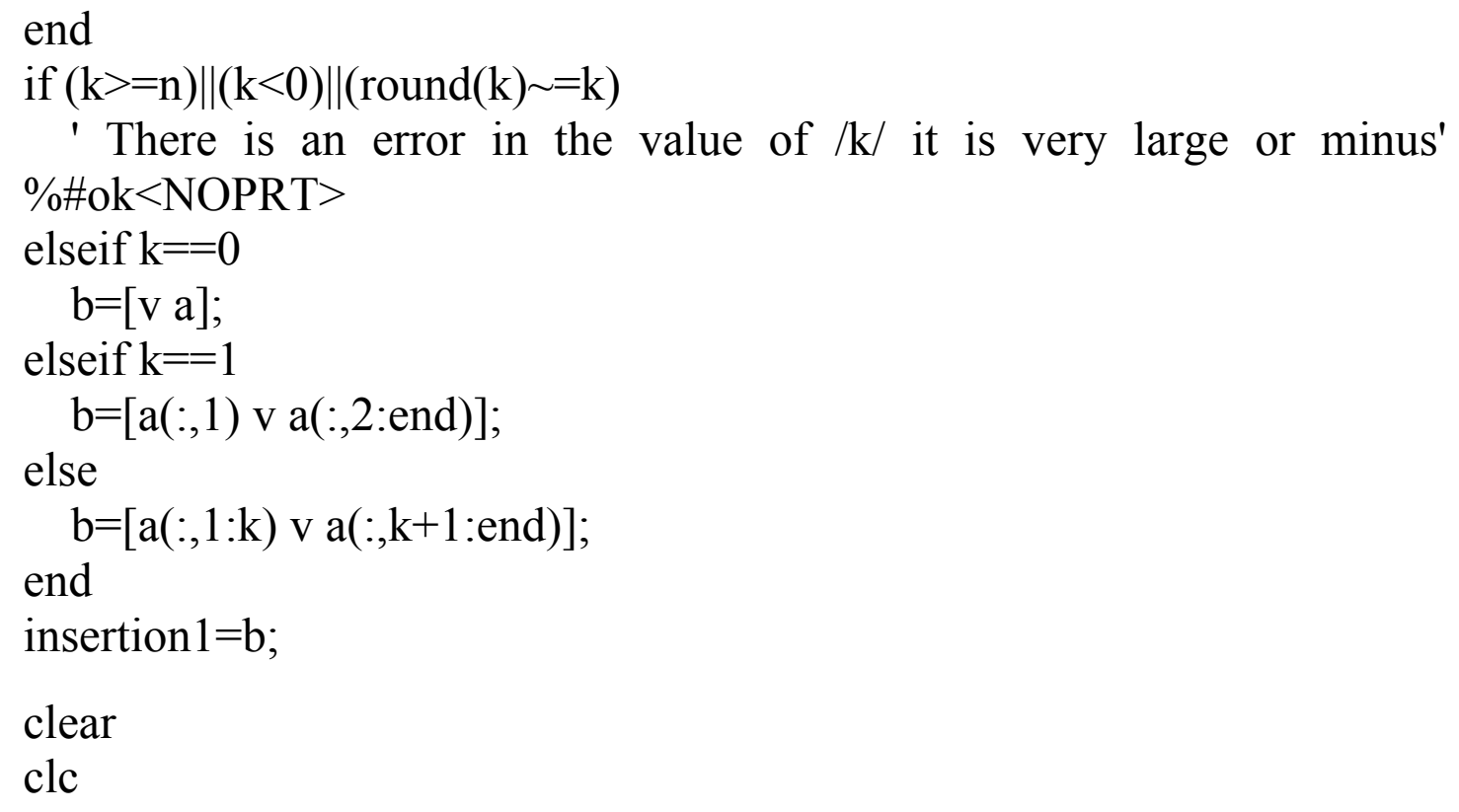

\%First Point $\mathrm{Mu}$ is the input series

\%Type the value of $/ \mathrm{Mu} /$ here

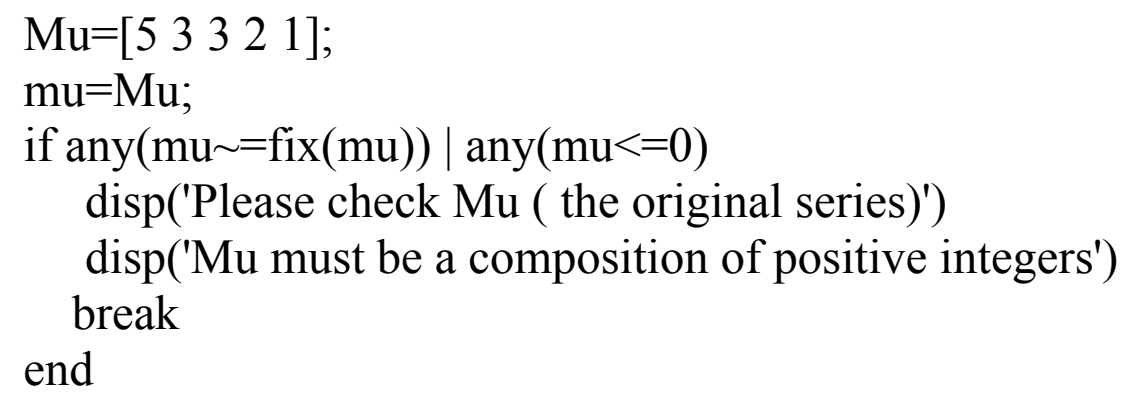

\section{\%Third Point /b/}

\%Type the value of $/ b /$ here

$b=7$;

if $b<$ length $(\mathrm{mu})$

$\operatorname{disp}($ 'Please check /b/')

$\operatorname{disp}\left(1 / \mathrm{b} /\right.$ must be $>=$ the number elements of $\left./ \mathrm{Mu} /{ }^{\prime}\right)$

break

end 
\%Forth Point Beta_Numbers /b_num/

residue $1=$ zeros $(1, \mathrm{~b}$-length $(\mathrm{mu})$ );

b_num $=[$ mu residue 1$]+b-[1: b]$;

$\%+\mathrm{H}+\mathrm{H}+\mathrm{H}+\mathrm{H}+\mathrm{H}+\mathrm{H}+\mathrm{H}+\mathrm{H}$ Begin

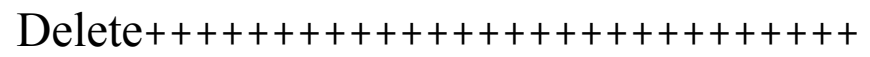

\%for example please, to delete the following b_num

$\%$ b_num $=\left[\begin{array}{lll}0 & 2 & 5\end{array}\right]$;

$\%$ b_num $=\left[\begin{array}{llllll}11 & 8 & 7 & 5 & 3 & 1\end{array}\right]$

$\%+1+1+1+1+1+1+1+1+1+1+$ Hnd

to

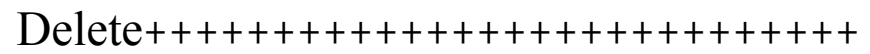

\%Fifth Point /e/

\%Type the value of /e/ here it must be $>1$

$\mathrm{e}=2$;

if $(\mathrm{e}<=1) \mid(\mathrm{e} \sim=$ fix $(\mathrm{e}))$

$\operatorname{disp}(' / \mathrm{e} /$ must be a positive integer number $>=2$ ')

break

end

\%Step 5 to make the table called here /E_Pure /

\%depending on the /b_num/ and the /e/.

$\%$ to put the numbers in the series /b_num into the matrix /E_Pure/

$\% \quad\left[\begin{array}{lllll}0 & 1 & 2 & \ldots & (\mathrm{e}-1)\end{array}\right.$

$\%$ e e+1 e+2 ... 2e-1;

$\% \quad \ldots \quad$ ]

bmax $=\max \left(b \_n u m\right)$;

$\% \mathrm{D}$ is the length of the new matrix

$\mathrm{d}=$ fix $(($ bmax $) / e)+1$;

$\%$ /Big_Value/ used to demonstrate the free positions from $/ \mathrm{m} 1 /$

Big_Value $=1000000$;

$\mathrm{C} 1=$ ones $(\mathrm{d}, \mathrm{e}) *$ Big_Value;

$\% \%$ function

$\% \%$ bnum is the series $\left(1 *^{*} n\right)$

$\% \% \mathrm{C} 1$ is the corresponding Matrix for it 


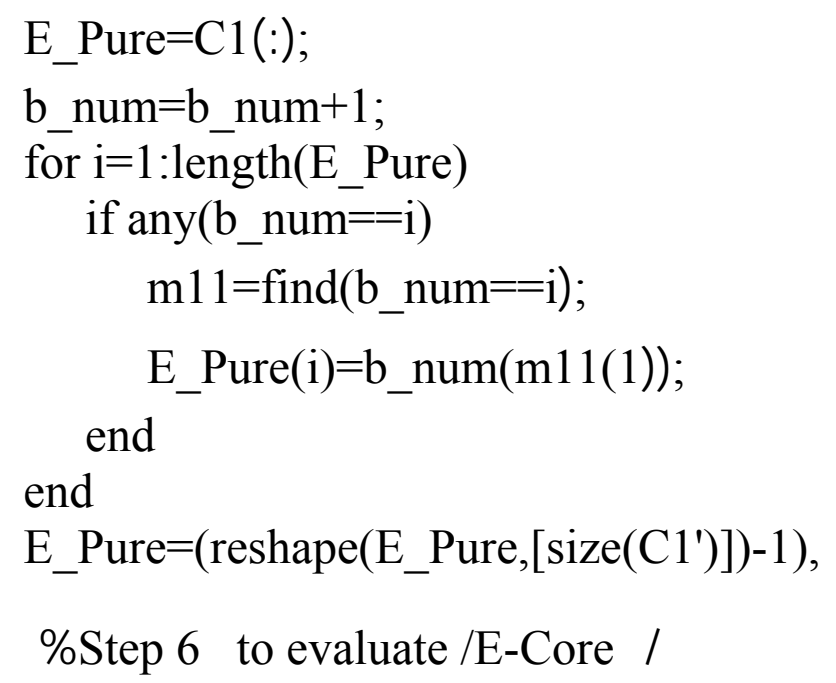

$\%$ Now we must make the balls go up to fill each empty space $\mathrm{m} 2=$ E_Pure;

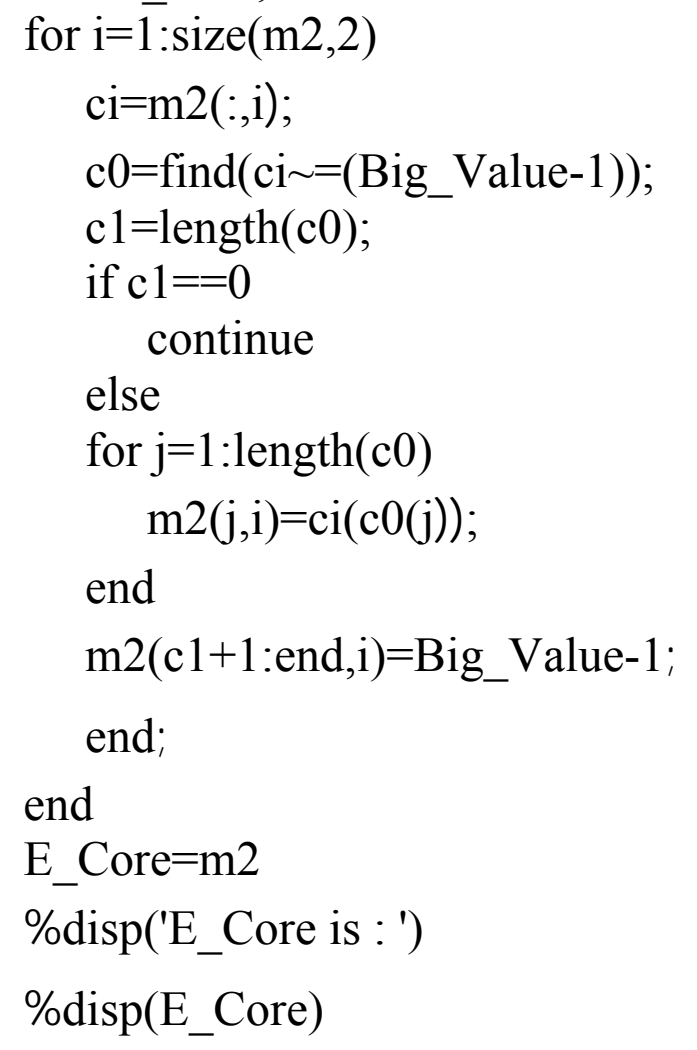

$\% \operatorname{disp}('$

\%disp(' Here we will make EXPANSION to our Matrix /E_Pure/ ')

\%function [Expansions]=expansion(E_Pure,Big_Value,e)

\%First we must check for the right positions technique 
\%that is /accepted_x_position/ and /accepted_y_position/

pqpq $=1$;

ddd $=$ size(E_Pure);

$\mathrm{d} 1=\mathrm{ddd}(1) ; \mathrm{d} 2=\mathrm{ddd}(2)$;

great_x_value $=1$;

great_y_value $=1$;

for $\mathrm{i}=1: \mathrm{d} 1$

for $\mathrm{j}=1: \mathrm{d} 2$

if $E \_$Pure $(i, j) \sim=($ Big_Value -1$)$

great_x_value $=\mathrm{i}$;

great_y_value $=\mathrm{j}$;

end

end

end

if great_y_value $==\mathrm{d} 2$

accepted_x_position $=$ great_x_value +1 ;

accepted_y_position $=1$;

else

accepted_x_position $=$ great_x_value;

accepted_y_position $=$ great_y_value +1 ;

end

our_balls=length(find(E_Pure $=($ Big_Value-1) $))$;

$\mathrm{b}=$ our_balls;

\%Now we must evaluate the equation : $\mathrm{b}+\mathrm{k}=\mathrm{c} * \mathrm{e}+\mathrm{d}$

\%here we call the value of all possible solutions by /may_be_roots/

may_be_roots $=$ ];

for $\mathrm{k}=0$ :accepted_x_position*e+e-1-b

for $\mathrm{d}=0: \mathrm{e}-1$

for $\mathrm{c}=0$ :accepted_x_position

if $(\mathrm{b}+\mathrm{k})==(\mathrm{c} * \mathrm{e}+\mathrm{d})$

end

may_be_roots $=[$ may_be_roots; $[\mathrm{b} \mathrm{k} \mathrm{c} \mathrm{e} \mathrm{d}]$;

\footnotetext{
end

end

end

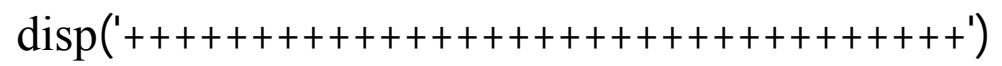

$\operatorname{disp}($ ' We can insert /c/ points at the left of the column /d / ')

$\operatorname{disp}(' \quad$ All Solutions for our E_Pure are : ')

$\operatorname{disp}\left(' \quad b+k=c * e+d^{\prime}\right)$
} 


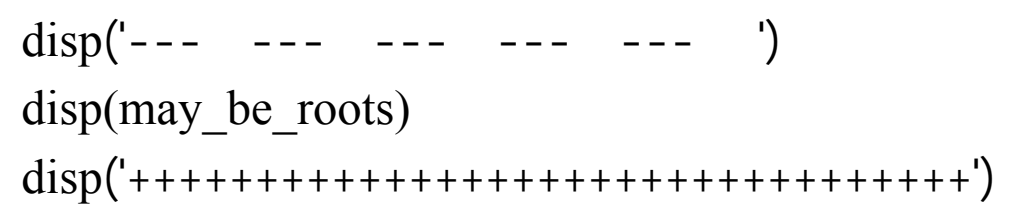

\%insert_column as the rows of /may_be_roots/ for the matrix /E_Pure/

$\%$ function $\mathrm{z}=$ Check_Insertion(E_Pure,Big_Value,may_be_roots)

$\mathrm{ddd}=$ size(E_Pure);

$\mathrm{d} 1=\mathrm{ddd}(1) ; \mathrm{d} 2=\mathrm{ddd}(2)$;

great_x_value $=1$;

great_y_value $=1$;

for $\mathrm{i}=1: \mathrm{d} 1$

for $\mathrm{j}=1: \mathrm{d} 2$

if $E \_$Pure $(i, j) \sim=($ Big_Value -1$)$

great_x_value $=\mathrm{i}$;

great_y_value $=\mathrm{j}$;

end

end

end

if great_y_value $==\mathrm{d} 2$

accepted_x_position $=$ great_x_value +1 ;

accepted_y_position $=1$;

else

accepted_x_position=great_x_value;

accepted_y_position $=$ great_y_value +1 ;

end

Origion_E_Pure=E_Pure;

for $\mathrm{i}=1: \overline{\mathrm{d}} 1$

for $\mathrm{j}=1: \mathrm{d} 2$

if E_Pure $(\mathrm{i}, \mathrm{j}) \sim=($ Big_Value-1)

$\%$ here $/ 1 /$ means there is an origion ball in this position

Origion_E_Pure $(i, j)=1$;

else

$\%$ here $/ 0 /$ means that there is nothing ball in this position Origion_E_Pure $(\mathrm{i}, \mathrm{j})=0$;

end

end

end

if great_y_value $==\mathrm{d} 2$

$\mathrm{w}=\mathrm{zeros}($ size(Origion_E_Pure, 2$))$;

$\mathrm{w}=\mathrm{w}(1,:)$;

Origion_E_Pure $=[$ Origion_E_Pure;w]; 
end

Origion E Pure \%\#ok $<$ NOPTS $>$

solutions(pqpq).E_Pure=Origion_E_Pure;

solutions $(p q p q) . e q u a t i o n=z e r o s(\operatorname{size}($ may_be_roots $(1,:)$;

\%Now we will simulate the case of all solutions

\%at first we must evaluate the vector/c4/ that will insert it in the

\%matrix Origion_E_Pure to make all solutions may be possible.

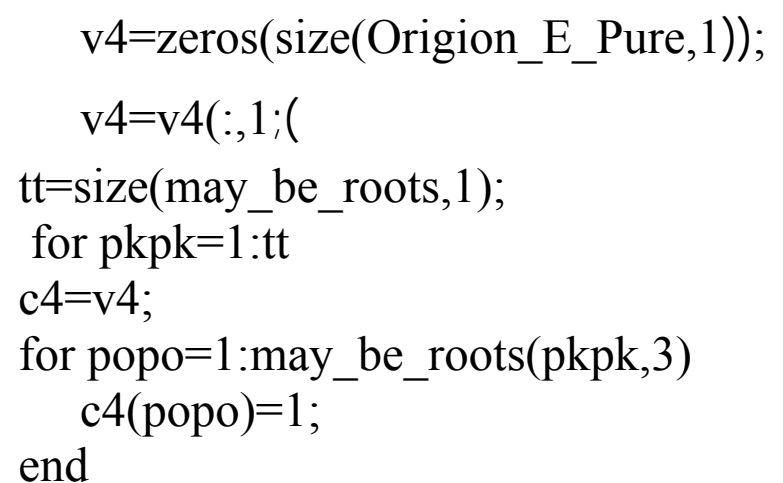

may_be_matrix=ins_column(Origion_E_Pure,c4,may_be_roots(pkpk,5)); $\mathrm{s}(\mathrm{pkpk}) . \mathrm{m}=$ may_be_matrix;

new_matrix $=$ check_acception_matrix(may_be_matrix,accepted_x_positi on, accepted_y_position);

if new_matrix $==0$

continue

else

$$
\text { pqpq }=\text { pqpq }+1 \text {; }
$$

solutions(pqpq).E_Pure=may_be_matrix;

end

solutions(pqpq).equation=may_be_roots(pkpk,:)

end

clc

solutions(1:end).E_Pure

This research attempt to find "radical ( $\mathrm{s})$ " from a given tree. That is to go back to this tree's base.

\section{Rule (3.2):}

To find the radical (s), we'll follow:

1) Count the beads from the given tree which we attempt to find its radicals, and let it denote by $b$.

2) Sorting the runners in the form below:

runner 1 by -1

runner 2 by 0 


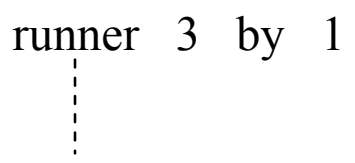

runner e by e-2

3) Let the number of runner is $\mathrm{e}^{\prime}=\mathrm{e}-1$.

4) Obviously, the last runner and the runners which hold $\beta_{1}$, they will not be discarded.

5) We note the number of runners which are full up with beads without any spaces by $t_{0}$, and $b^{\prime}=b-t_{0}$.

6) Applying Fayers's rule: $b^{\prime}+u=t_{0} e^{\prime}+d^{\prime}, 0 \leq d^{\prime} \leq e^{\prime}-1$, if a value found that carry out the above equation by deleting from the beads and then delete the runner, our reduction is succeed. Otherwise, this is "sterile" or "useless" and will be neglected. Then, we seek another runner having the same beads feature $t_{1}$ and apply the same previous step: $b^{\prime}+K=t_{1} e^{\prime}+d^{\prime}$.

7) repeat step (6) and neglect all sterile cases, and continue with the useful one. Then apply all steps from (1) to (6) on it.

8) When there is no case can keep on with it, this means all cases are steriles. This case considers the radical for the given tree.

For example :

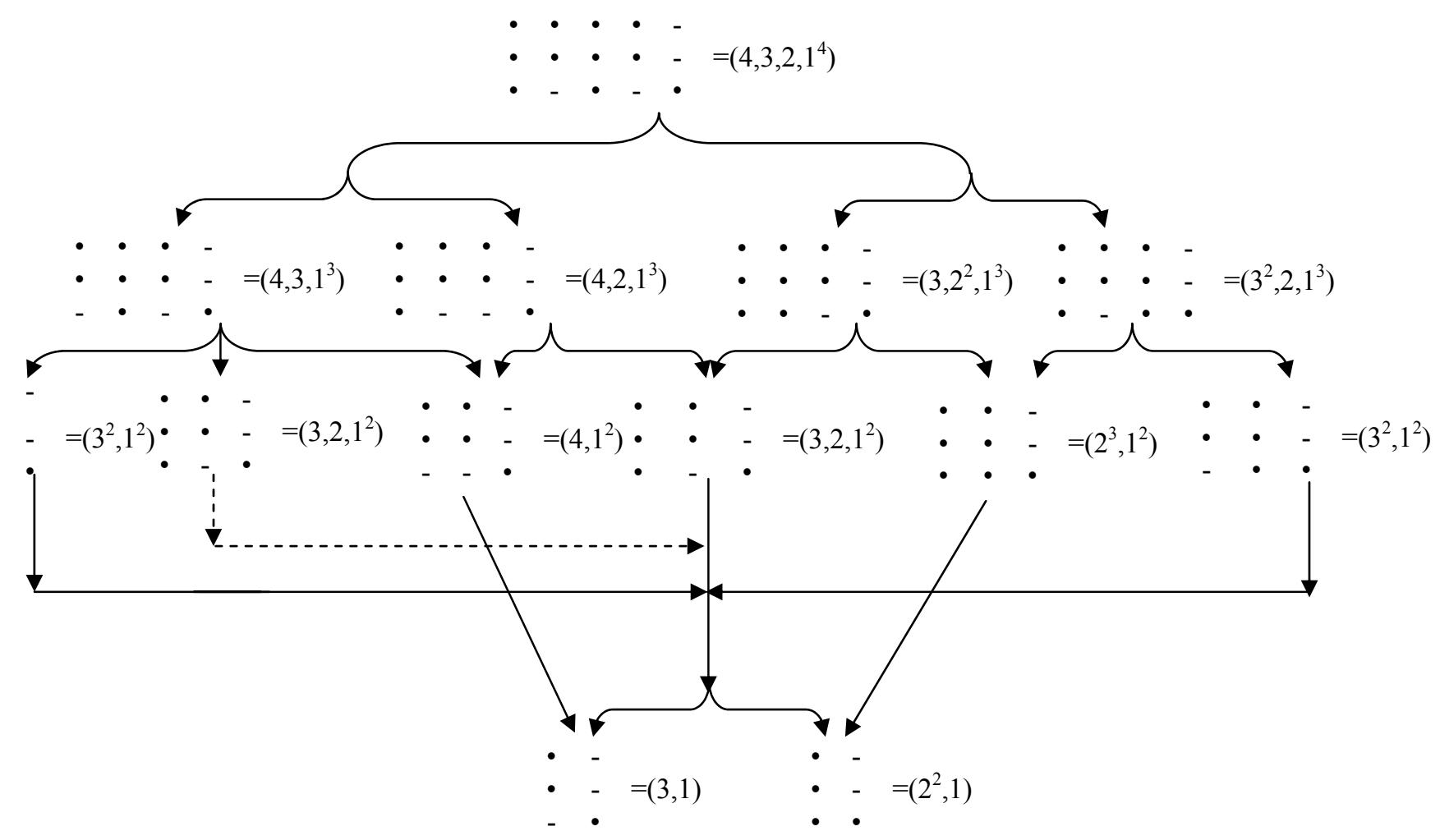

Then the radicals of this example are $(3,1)$ and $\left(2^{2}, 1\right)$. 


\section{References:}

1) R. Dipper and G. James, Representations of Hecke algebras of general linear groups. proc. London Math. Soc., no. 3, 52 (1986), 20-52.

2) M. Fayers, Weight two blocks of Iwahori-Hecke algebra in characteristic two, Math. Cambridge philos. Soc. 139 (2005), 385397.

3) M. Fayers, Irreducible Specht modules for Hecke algebra of type A, Adv. Math., 193 (2005), 438-452.

4) M. Fayers, Another runner removal theorem for r-decomposition numbers of Iwahori-Hecke algebra and q-Schur algebra, J. algebra 310 (2007), 396-404.

5) M. Fayers, An extension of James's conjecture, Int. Math. Research Notices, (to appear).

6) M. Fayers and K. M. Tan, Adjustment matrices for weight three blocks of algorithms of Iwahori-Hecke algebra, J. algebra 306 (2006), 76-103.

7) G. James, Some combinatorial results involving Young diagrams, Math. Proc. Camb. Phil. Soc., 83 (1978), 1-10.

8) G. D. James, S. Lyle and A. Mathas, Require blocks, Math. Z. 252 (2006), 511-531.

9) A. Mathas, "Iwahori- Hecke algebras and Schur algebras of the Symmetric Group", University lecture series15, Amer. Math. Soc., 1999. 